\title{
Cet autre qui me regarde. Phénoménologie de la mémoire et temps eschatologique dans Le Premier Jardin et Oh les beaux jours
}

STÉPHANE INKEL

UNIVERSITÉ QUEEN'S

Résumé. Alors que Les fous de Bassan s'attachait à mettre en scène une Amérique métonymique tournée vers sa fin, tout se passe comme si Le premier jardin visait pour sa part à lester cette Amérique incertaine et eschatologique par l'anamnèse de son commencement, qui, chez Anne Hébert, prend le plus souvent la forme d'un re-commencement. II s'agira ici d'interroger le dispositif temporel mis en fiction dans le roman, notamment par l'entremise d'une lecture de la pièce Oh les beaux jours et de sa temporalité figée d'après-coup. À travers cette analyse de la temporalité, on voudra également mettre en relief l'instrumentalisation de l'histoire collective par le sujet, qui cherche ainsi à se prémunir de sa propre mémoire, constamment mise à distance au sein du roman.

Mots-clés : Mémoire, Temps eschatologique, Phénoménologie de la mémoire, Samuel Beckett, Commencement.

Alors que Les fous de Bassan s'attachait à mettre en scène une Amérique tournée vers sa fin, et littéralement hantée par ce qui occupe l'écart entre la mort réelle des cousines Atkins et la mort symbolique de la communauté métonymique de Griffin Creek, tout se passe comme si Le premier jardin visait pour sa part à lester cette Amérique incertaine et eschatologique de l'anamnèse des premiers temps de la coIonie. Mais est-ce bien différent? À suivre les méandres des discours intérieurs des personnages de I'un et l'autre romans, force est de constater que ce qui fait office de représentations de l'origine concourt à les mettre à l'abri de cette fin pressentie, eux qui ont en commun de recourir aux artifices d'une histoire monumentale («galerie des ancêtres », projet de théâtre historique, etc.) afin de repousser le moment de remémoration de l'événement qui les occupe. Tous deux romans de l'anamnèse, ils ont ainsi en commun d'entremêler une certaine tonalité eschatologique avec des motifs du recommencement, tout en articulant les données mémorielles d'une col- 
lectivité avec l'aventure d'une subjectivité. Si je fais intervenir la notion lacanienne des « deux morts», récemment commentée par le philosophe Slavoj Žižek, c'est précisément parce qu'elle permet de lier la mort comme destin individuel au statut d'une société dans son ensemble par l'entremise de l'ordre symbolique qui la fonde. Or, c'est cette difficile articulation entre la mémoire du sujet et la mémoire collective, notamment recueillie à travers I'histoire, qui s'offre comme une énigme à déchiffrer au lecteur du Premier jardin.

Pour le dire vite, ce qui permet de distinguer la mort réelle de la mort symbolique, c'est « le règlement des comptes » (Žižek, 2011 : 331) - et donc le paiement d'une certaine dette symbolique. La mort symbolique, dans ce premier cas de figure, correspond ainsi au lent travail du deuil, indispensable à la poursuite de nouvelles visées. Mais dans un deuxième sens, emprunté par Lacan au discours du Pape qu'on trouve dans l'immense Histoire de Juliette de Sade, elle signifie surtout l'anéantissement de l'ordre symbolique lui-même, c'est-à-dire tout ce qui fonde l'univers du sujet, ce qui l'apparente à la pulsion de mort dans la mesure où celle-ci trouve son point d'appui dans le travail du signifiant : « [I]l est en effet exigible que ce dont il s'agit [avec ce concept] soit articulé comme pulsion de destruction, pour autant qu'elle met en cause tout ce qui existe. Mais elle est également volonté de création à partir de rien, volonté de recommencement. » (Lacan, 1986 : 251) La visée d'une telle abolition de la mémoire serait donc une possibilité de recommencement, un nouveau départ ou un nouveau masque ajusté au visage du sujet, comme le laisse d'ailleurs entendre Stevens Brown, dans la lettre qui clôt Les fous de Bassan : une fois le travail de remémoration comme « règlement des comptes » mis en branle, la mort symbolique de Griffin Creek semble enfin permettre I'apparition d'autre chose : « Le monde vire sur sa quille et recommence. » (Les fous de Bassan, 1982 : 236)

Si le « premier jardin » qui donne son titre à ce roman de 1988 évoque à la fois le lieu du tout premier Commencement (ou Bereshit, d'après le premier mot de la Torah, qu'on a traduit par «Genèse »), de même que celui créé à son image par «le premier homme » et « la première femme » (Le Premier Jardin, $1988: 76$ ) du Nouveau Monde qui avaient pour nom Louis Hébert et Marie Rollet, c'est encore une fois pour souligner l'importance de la répétition, cette occasion de re-commencer, qui, comme on le sait, joue toujours un rôle primordial dans les textes d'Anne Hébert ${ }^{1}$. Grâce à ce jardin, suivant le récit de Raphaël, «[t]oute l'histoire du monde s'est mise

$\overline{1 .}$ C'est la thèse fondamentale du livre de Daniel Marcheix, qui voit dans chaque texte hébertien le recommencement d'une différentiation manquée dès I'origine (Marcheix, 2005). 
à recommencer à cause d'un homme et d'une femme plantés en terre nouvelle. » $\left(P J^{2}: 77\right)$ Mais le commencement, suivant le palimpseste biblique toujours repérable dans la trame du texte hébertien, c'est avant tout la lettre; signifiant ou adresse en provenance de l'autre. Deux lettres, en ouverture du roman, « décident de son retour [celui de Flora] au pays natal » ( $P J: 9)$ : un appel de Maud, sa fille, et la proposition de jouer Winnie dans Oh les beaux jours du directeur du théâtre l'Emérillon.

Dès cette première page, et à partir du titre, se trouvent donc convoqués par le roman les textes, biblique et beckettien, sur lesquels il va chercher à articuler la question de la mémoire, individuelle, à celle de l'histoire et à l'expérience du temps qu'elle implique. Arrêtons-nous d'ailleurs un instant sur cette autre figure contenue dans la lettre : I'Emérillon³. Certes, voilà une figure du passé engageant l'imaginaire du Nouveau Monde et présidant de son autorité à la nouvelle «traversée » de Flora Fontanges. Mais se souvient-on qu'un « émerillon » est aussi un dispositif d'attache? Quel raccord cherche-t-on à lui faire effectuer entre cet imaginaire du Nouveau Monde qu'il porte avec lui et l'imaginaire beckettien qu'il introduit par le théâtre qu'il désigne? Il s'agira ici d'interroger le dispositif de lecture instauré par Le premier jardin, en particulier la double temporalité induite par les textes biblique et beckettien, entre re-commencement mythique ou historique et temps eschatologique. Je chercherai également à préciser le sens du recours à I'histoire et ce qu'il met en œuvre dans l'entreprise de remémoration sans cesse différée conduite par le roman.

\section{Temporalité beckettienne}

A priori, peu de choses permettent de rapprocher les œuvres de Samuel Beckett et d'Anne Hébert. Si le premier opère, à partir de ses premières Nouvelles écrites directement en français, autour de 1945, une réduction progressive de la représentation du référent, jusqu'au point limite de L'innommable, pure recension des conditions d'énonciation du sujet, la seconde a toujours situé ses textes dans un univers référentiel parfaitement reconnaissable et dûment historicisé. Comment lire, dès lors, la référence à Oh les beaux jours que I'on retrouve à la fois dans l'incipit et dans la dernière page du Premier jardin? Trois éléments de la pièce semblent trouver un écho dans le roman. Le premier, le plus évident, concerne la théâtralité, le caractère indispensable du jeu pour l'identité précaire de Flora Fontages, dramaturge et comé-

2. PJ pour Le premier jardin, 1988.

3. L'Emérillon est non seulement l'un des trois navires de l'expédition de 1535, c'est aussi avec lui que Cartier remonte le fleuve jusqu'au lac Saint-Pierre, en route vers Hochelaga. 
dienne de sa vie, comme l'anamnèse patiente du roman le montrera. Mais si cette identité s'est construite par strates superposées, succession de masques, elle a aussi opéré cette succession à coup d'effacements répétés, d'oubli, ce qui rapproche sa mémoire de celle, pleine de trous, de Winnie.

Sans être aussi central dans la pièce qu'il l'est pour le roman, cet enjeu de la mémoire constitue le deuxième élément, malgré des différences importantes, rapprochant les deux œuvres. À cet égard, il est bon de rappeler la déliquescence dont elle fait l'objet dans cette pièce où, à l'égal de la mémoire défaillante de Pozzo ou d'Estragon, dans En attendant Godot ${ }^{4}$, elle se dilue dans la stase d'une temporalité d'après-coup qui semble appelée à s'étirer indéfiniment, finissant par atteindre le langage lui-même ${ }^{5}$. Mais si d'un côté la mémoire est trop lourde de souvenirs propres à ramener le sujet sur les lieux d'un trauma refoulé, et donc toujours sur le point de faire retour, alors que de l'autre, elle semble tout juste bonne à pouvoir identifier les protagonistes et à distinguer un avant de l'instant présent, il faut voir qu'une telle opposition ne fait que reconduire les effets de dispositifs temporels également fort distincts.

Toutefois, l'élément emprunté de la pièce beckettienne qui semble le plus déterminant concerne le rôle du regard imparti à l'autre, ici indispensable à la parole du sujet. Rappelons le dispositif d'Oh les beaux jours, qui se verra lui-même radicalisé dans Pas moi, qui n'accompagne pas sans raison la première pièce dans la même édition, chez Minuit, depuis 1974. Winnie, la protagoniste principale de la pièce, est ensevelie dans un monticule de sable jusqu'à la taille, ce qui la prive de mobilité et met par le fait même l'accent sur la parole comme seule modalité de l'« action » dramatique. Mais pour que cette parole puisse fonctionner, c'est-à-dire occuper de son babil rituel et factice le temps immobile scandé par le son du réveil, la présence de

4. On se souviendra que la conclusion de la pièce justifie cette disparition de la mémoire par le jeu de la répétition qui enchaîne les personnages à leur place et, corrélativement, par la temporalité distendue de l'instant: « Vous n'avez pas fini de m'empoisonner avec vos histoires de temps? C'est insensé! Quand! Quand! Un jour, ça ne vous suffit pas, un jour pareil aux autres il est devenu muet, un jour je suis devenu aveugle, un jour nous deviendrons sourds, un jour nous sommes nés, un jour nous mourrons, le même jour, le même instant, ça ne vous suffit pas? Elles accouchent à cheval sur une tombe, le jour brille un instant, puis c'est la nuit à nouveau. » (Beckett, $1989: 126)$

5. Voir, par exemple, l'épisode où Winnie tente de lire - et comprendre - l'inscription sur sa brosse à dents, finissant par buter sur le mot « soie de porc » : QQu'est-ce que c'est au juste, un porc? (Un temps. De même.) Une truie, ça oui, évidemment, je sais, mais un porc? » (Beckett, $1986: 25$ ) 
I'autre s'avère indispensable ${ }^{6}$. La fonction de Willie, dont la parole est aussi occasionnelle qu'impersonnelle - lui qui se limite à lire des extraits d'un journal ayant survécu à la catastrophe indéterminée ayant fait du monde représenté un pur espace d'aprèscoup, ou à répondre, le plus succinctement possible, aux questions de Winnie -, se limite en effet à son regard, à sa capacité d'authentifier l'existence de celle qui n'est plus que parole tournant à vide; un regard qui n'a même pas besoin d'être perçu, mais posé à titre d'hypothèse'. Structuralement, donc, cette présence de l'autre est indispensable à la poursuite de la comédie qui consiste à se regarder dans le miroir, à faire l'inventaire de ses possessions, et à relater des souvenirs qui ne portent nullement à conséquence ${ }^{8}$. C'est d'ailleurs cette structure minimale du sujet qui a donné naissance à l'une des images scéniques les plus saisissantes de Beckett, dans Pas moi : celle d'une simple «bouche » dévidant un discours haletant et hachuré en présence d'un « auditeur » discret et silencieux, limité à un seul geste (« une sorte de haussement des bras dans un mouvement fait de blâme et de pitié impuissante » [Beckett, $1986: 95])$, répété à quatre reprises.

Les trois éléments retenus par Anne Hébert étant identifiés, il convient de relever de manière un peu plus précise les emprunts directs à la pièce de Beckett repérables dans les différentes allusions qui y sont faites à travers le roman. La première chose qui frappe le lecteur de Beckett, c'est la transformation qu'opère Flora Fontages à la physiologie de Winnie, au moment où elle en convoque l'image lors de sa traversée de I'Atlantique : « [Elle] appelle Winnie de toutes ses forces. Fait venir une très vieille femme en elle. La dévisage, l'observe, l'épie. Se confronte à elle. Essaie de lui ressembler. » $(P J: 11)$ Pour ce faire, elle entend couper ses pointes rousses et blondes jusqu'à la repousse « en vue de se faire la tête grise de Winnie. » ( $P J: 12)$ Plus tard,

6. C'est ce qui différencie, ultimement, l'expérience théâtrale des textes narratifs de l'écrivain irlandais, où le soliloque d'une conscience limitée à sa seule «tête dite siège de tout » (Beckett, 1991) ne requiert pas cette présence de l'autre dans sa matérialité, au contraire de la scène. Même La dernière bande matérialise cet autre que représente le sujet pour lui-même à dix ans de distance par la matérialité de la bande sonore (et l'objectalité de la voix qu'elle produit) écoutée par Krapp à chaque anniversaire.

7. Lors du deuxième acte, par exemple, lorsque Winnie est enterrée jusqu'au cou, qu'elle ne peut plus se tourner en direction de son compagnon, et qu'elle ne parvient plus à l'entendre, elle posera la condition minimale de cette présence de l'autre : « [N]e pas savoir de façon certaine » qu'il n'est plus. Voir Beckett, 1986 : 62.

8. L'on pourrait comprendre ainsi le regard d'effroi de Winnie interprétée par Andrée Lachapelle dans la mise en scène d'André Brassard, à l'automne 2008, lorsque Willie rampe jusqu'au revolver juste à la droite de sa tête (I'ambiguïté de la visée du geste est maintenue dans le texte par la célèbre réplique : «C'est moi que tu vises, Willie, ou c'est autre chose? » [Beckett, 1986 : 75]). La perspective qui est source d'effroi n'est certes pas d'être «visée » par le révolver, mais de continuer seule devant l'éternité après que l'autre a renoncé. 
lors des premières répétitions, elle l'évoque même comme une « petite vieille ratatinée et muette » $(P J: 46)$; il s'agit, en somme, de faire de Winnie l'incarnation de la vieillesse et de la mort qui approche, voire du caractère « délétère » $(P J: 187)$ de la chair, comme le précise la narration dans la description de la représentation qui met fin au roman. Pourtant, la didascalie de départ indique tout autre chose, même si l'on peut penser qu'Anne Hébert se référait davantage à la prestation de Madeleine Renaud, qui a créé le rôle en 1963 et l'a interprété jusqu'en 1986, qu'aux indications de Beckett : «Winnie. La cinquantaine, de beaux restes, blonde de préférence, grassouillette, bras et épaules nus, corsage très décolleté, poitrine plantureuse, collier de perles ${ }^{9}$. » (Beckett, $1986: 11-12$ ) Or, ce n'est pas là un détail. Alors que Beckett insistait sur la paralysie d'un temps d'après-coup, à la fois post-apocalyptique et intériorisé, Anne Hébert, dans sa relecture, insiste plutôt sur la proximité de la mort que les personnages réclament - représentée notamment par l'attrait du pistolet «browning » auprès du couple -, et sur la déréliction qui frappe l'identité et la mémoire des personnages. Pourquoi une telle différence d'accents? Tout se passe comme si Anne Hébert était sensible à la répétition présente dans le théâtre beckettien, similaire à la structure psychique de Flora Fontanges, tout en voulant en souligner la fin possible par la mort. En d'autres termes, en substituant un corps en décrépitude aux « beaux restes » de Winnie, pour reprendre l'expression consacrée, ceux-là même que Flora Fontanges se plaît à exhiber face à l'amant de sa fille, Anne Hébert souligne la prégnance de la fin, dans Oh les beaux jours, mais tout en mettant de côté le temps figé qui en forme pourtant le ressort. On ne saurait pourtant faire intervenir innocemment le paysage eschatologique mis en scène par la pièce de Beckett dans un roman où I'histoire joue le premier rôle.

\section{La force « qui retient » le temps et les souvenirs}

Cette fascination de Flora pour la déréliction du corps et la proximité de la mort, dans Oh les beaux jours, ressemble donc à première vue à un contresens de lecture. Mais rien n'est simple, chez Beckett, où les images créées pour la scène sont souvent la représentation de la structure même du sujet, comme le précise Žižek dans son commentaire de Pas moi, où il s'agissait de «visualiser/matérialiser cet emplacement structurel en une figure dans l'espace imaginaire de la scène ». (Žižek, $2010: 150$ ) Explorations du dedans, du «crâne », ou de la «cloison » de mots qui séparent le 
dedans du dehors, comme le dit le sujet de L'innommable, les textes de Beckett n'en offrent pas moins une représentation du temps perçu dans certaines conditions, faisant de cette façon écho à une certaine schématisation du temps de I'histoire. Dans son théâtre, il se passe cette chose très curieuse que l'expérience temporelle qu'il donne à percevoir, voire à expérimenter, de pair avec les personnages, tient avant tout du dernier délai, de la Fin de partie aux derniers jours, malgré le (ou grâce au) fait que quelque chose est déjà survenu. Il est toujours déjà trop tard, même si les différents personnages ne cessent de réclamer une fin définitive du temps pouvant enrayer la répétition : «Ça ne va donc jamais finir ! » (Beckett, 1957 : 38), se plaint Hamm, alors que Winnie se réjouit plutôt de la sorte de paralysie du temps absolument vide qu'il lui faut désormais meubler par sa routine obsessionnelle habitée par de rares lambeaux de souvenirs : «Quelle bénédiction que rien ne pousse, imagine-toi si toute cette saloperie se remettait à pousser. »; «Très étrange. (Un temps.) Jamais rien qui change. »; «Peut-on parler encore de temps ? » (Beckett, $1986: 41$, $53,60)$ En fait, le temps n'aura sans doute jamais été aussi présent en tant que représentation, dans la mesure où l'absence d'action proprement dramatique ne laisse rien d'autre que lui à percevoir pour le spectateur; on le sent, il nous traverse, et pourtant, il n'est plus agissant sur ces figures qui apparaissent comme autant de variations des figures du Purgatoire qu'affectionnait particulièrement Beckett.

Or, s'il y a une constante, dans l'univers beckettien, c'est bien ce désir d'en finir une fois pour toute qui se voit constamment refusé, la Fin de partie se donnant toujours à voir dans ses derniers soubresauts pourtant indéfiniment répétés, qui en appellent à d'autres textes, Pour finir encore et peut-être pouvoir dire, comme le narrateur de Malone meurt : « Je serai quand même bientôt tout à fait mort enfin. » (Beckett, 1995 : 7) Mais ce qu'il faut bien voir, c'est que cet appel répété envers une fin qui ne vient pas indique précisément qu'elle ne saurait venir, que la structure temporelle qui prévaut dans cet univers fictionnel l'en empêche, marquant peut-être par là une panne d'événementialité faisant écho à la stupeur qui fait suite aux événements du milieu du siècle dont Beckett aura été un témoin privilégié en tant qu'acteur de la Résistance. Or, en maintenant suspendu ce temps que tous ses personnages voudraient voir disparaître, Beckett se trouve à offrir l'image inversée d'une conception théologique du temps ayant longtemps soutenu l'édifice occidental de l'histoire, conception que l'on retrouve synthétisée de la manière la plus forte par le juriste allemand Carl Schmitt, qui en emprunte la formulation à une figure bien précise des Épîtres de Paul : 
Le concept décisif qui fonde historiquement sa continuité est celui de la puissance qui retient [Aufhalter], du kat-echon. Empire signifie ici la puissance historique qui peut retenir l'apparition de l'Antéchrist et la fin de l'ère actuelle [...] Je ne crois pas qu'une autre représentation de I'histoire que celle du kat-echon soit même possible pour une foi chrétienne originaire. La foi en une force qui retient la fin du monde jette le seul pont qui mène de la paralysie eschatologique de tout devenir humain jusqu'à une puissance historique aussi imposante que celle de l'Empire chrétien des rois germaniques (Schmitt, $2001: 64$ ).

L'argument de Schmitt, qui vise à justifier théologiquement l'immanence de l'action politique s'étant historiquement incarnée dans I'Empire chrétien (ou à travers I'institution de l'Église d'Occident), s'appuie ici sur un passage bien précis de la Deuxième épître aux Thessaloniciens :

Et vous savez ce qui le retient maintenant, de façon qu'il ne se révèle qu'à son moment. Dès maintenant, oui, le mystère de l'impiété [anomos] est à I'œuvre. Mais que seulement celui qui le retient soit d'abord écarté. Alors I'Impie se révélera, et le Seigneur le fera disparaître par le souffle de sa bouche, I'anéantira par la manifestation de sa Venue. (2 Th 2, 6-8)

Il va sans dire que l'ambiguïté du passage a donné lieu à de multiples interprétations tentant de savoir si cette étrange force «qui retient » doit être perçue de manière positive ou négative; négative, car elle empêche la Parousie, ou positive, comme le fait Schmitt, suivant une position déjà défendue par Tertullien dès le $2^{\mathrm{e}}$ siècle, car elle assure « la permanence du monde [...], la paix des choses [et] [...] le retard de la fin ». (cité par Agamben, $2000: 172$ ) Concept fondamental, donc, depuis ce Père de I'Église jusqu'au célèbre et décrié juriste, d'une théologie politique fortement conservatrice, le katechon paulinien a joué le rôle, pour notre tradition, d'une force temporelle (ou immanente) apparentée à I'histoire. Le penseur juif et historien des religions Jacob Taubes, grand lecteur de Paul, y va toutefois d'une remarque éclairante lorsqu'il réagit à l'usage de cette figure par Schmitt : «Carl Schmitt pense apocalyptiquement, mais d'en haut, à partir des pouvoirs ; moi je pense d'en bas. Mais nous partageons tous les deux l'expérience du temps et de l'histoire comme délai, comme dernier délai. Ce fut aussi, à l'origine, l'expérience chrétienne du temps. » (Taubes, 1999 : 164) Cette expérience messianique du temps «comme dernier délai » n'est évidemment pas sans lien avec la temporalité mise en œuvre dans les fictions aussi bien de Beckett que d'Anne Hébert. On se souvient de I'aphorisme énigmatique de Kafka selon lequel « [l]e Messie ne viendra que lorsqu'il ne sera plus nécessaire, il ne viendra qu'un jour après son arrivée, il ne viendra pas au dernier, mais au tout dernier jour. » (Kafka, 2001 : 105) L'expérience beckettienne du temps que I'on retrouve 
dans son théâtre semble se situer dans ce léger écart paradoxal entre le dernier et le « tout dernier jour », après la catastrophe mais dans l'attente de la fin dernière du temps, utilisant de manière parfaitement ironique cette force « qui retient » l'eschaton alors même que l'action n'y est plus nécessaire.

Si l'on revient à l'auteure du Premier jardin, on se souvient sans doute de la présence d'une autre figuration paulinienne de ce temps messianique dans Les fous de Bassan, où le pasteur Nicolas Jones médite sur le fait que «le temps se fait court ${ }^{10}$ » $\left(F B^{11}: 23\right)$, alors même que la communauté de Griffin Creek est comme suspendue entre les événements de 1936 et la « dispersion » définitive de ses familles fondatrices. À première vue, cet univers eschatologique propre au roman de 1982 semble étranger au roman qui suit, que la critique a pu lire comme l'énonciation poétique d'un «travail historiographique ». (Falardeau, 1997 : 563) Pourtant, l'apocalyptique peut être décelé en filigrane, non comme préfiguration de la fin, mais en tant qu'imaginaire enté à l'idée de recommencement, comme l'illustre parfaitement l'évocation de l'épisode des filles du Roi, chargée chacune d'incarner une parcelle d'une même entité, « Ève en personne [...], mais fragmentée en mille frais visages, [...] traversant I'océan, [...] pour nous sortir du néant ». (PJ : 99-100) Ce n'est d'ailleurs pas sans raison si la révolution a pu être décrite comme une forme sécularisée de l'apocalyptique, car au sein de l'une comme au sein de l'autre, la force destructrice n'est que la première de ses composantes, qui doit impérativement être suivie d'une « puissance formatrice de figures $\gg$. (Taubes, $2009: 12$ )

Sujet à l'identité maintes fois effacée puis forgée à nouveaux frais, Flora Fontanges renoue d'autant plus aisément avec l'utopie du recommencement propre à l'imaginaire du Nouveau Monde. Mais, par la même occasion, se pose la question du passage de cette logique étroitement identitaire, personnelle, à I'histoire, et ce, d'autant plus que celle-ci se voit recouverte immédiatement par les oripeaux du mythe, qui en est l'exacte négation. Pierre Nepveu a déjà posé la question de cette appropriation de I'histoire qu'implique le fait de se revendiquer du Nouveau Monde : «Pour que l'Amérique et le Québec américain deviennent un problème et un enjeu créateur, il a fallu que soit posée d'abord la question du sujet qui peut assumer et inventer ce Nouveau Monde. »(Nepveu, 1998 : 159) Soulignant le caractère mythique (et désincarné) des premiers mots de Mystère de la parole («Notre pays est à l'âge des premiers jours du monde » [Fuvre poétique, $1992: 62$ ]), Nepveu ajoute que c'est davantage

10. 1 Co $7,29$.

11. FB pour Les fous de Bassan, 1982. 
le paysage qui habite le sujet, l'envahit de son étrangeté irrémédiable (jusqu'à le conduire à la folie ou à la mort, comme dans « Le torrent ») que l'inverse. Quelle est la part du mythe; quelle est celle de I'histoire ? En quoi, dans Le Premier Jardin, I'un et l'autre visent-ils à « retenir » le passé à l'intérieur de soi, à empêcher le moment où «l'instant ne [nous] porte plus »? ( $P J: 123)$ Et si tous deux servaient à échapper au « temps comme délai » à proprement parler, et à ainsi rejoindre la stase temporelle qui est la quotidienneté de Winnie?

Ce qui a longtemps « retenu » l'eschaton et maintenu le monde dans son être, selon I'interprétation de Schmitt, c'est I'Église en tant qu'institution ayant relayé la force historique de l'Empire; en d'autres termes, le katechon s'incarne dans toute force prêtant une certaine pérennité à l'histoire. Il est intéressant de remarquer la prégnance lexicale du registre du « rempart » lorsqu'il s'agit de décrire la difficile confrontation du sujet hébertien avec sa mémoire; il devient dès lors encore plus frappant de constater que ce qui fait office d'un tel rempart contre le refoulé qui menace de faire retour, dans Le premier jardin, à la faveur des retrouvailles de Flora Fontanges avec la ville de son enfance, c'est précisément I'histoire - celle, particulière, de la « Nouvelle-France » et du nouveau commencement dont elle est I'occasion.

\section{Phénoménologie de la mémoire}

S'il y a une chose contre laquelle le sujet cherche à se prémunir, dans le roman, c'est, comme nous l'avons vu en introduction, sa propre mémoire. À ce titre, deux types d'obstacle se présentent pour lui barrer la route, qui tous deux nous ramènent à ce que nous avons dégagé de la pièce de Beckett : la présence de l'autre et le recours à I'histoire. Mais avant de repérer en détail l'usage qui en est fait, il convient de circonscrire le fonctionnement de la phénoménologie de la mémoire mise en œuvre par le roman.

Il peut à ce titre paraître utile de suivre la patiente relecture des textes de la tradition portant sur la mémoire et le souvenir effectuée par Paul Ricœur dans La mémoire, I'histoire, l'oubli, en particulier son « esquisse phénoménologique de la mémoire » (Ricœur, 2003 : 25-53), où il s'applique à suivre le trajet de la « remémoration » et le franchissement d'obstacles que cela suppose. Mais notons d'emblée le renversement qu'Anne Hébert fait subir à cette économie de la mémoire, du « Torrent » au Premier jardin, en passant par le rapport obsessionnel de Mme Rolland aux « remparts » dans Kamouraska. C'est que l'obstacle, saisi compulsivement par un sujet aux abois, vise 
toujours à repousser à plus tard, à garder indéfiniment à distance le retour du refoulé visant à faire coïncider le sujet avec le réel évanouissant qui le fonde. Il s'agit donc d'être attentif aux variations multiples de cette phénoménologie inversée (car visant à prévenir toute remémoration), gage du maintien d'une identité d'emprunt fondée sur la structure hystérique du « masque » de l'actrice.

Parmi les distinctions que Ricœur s'applique à dégager à travers la typologie qui ouvre son « esquisse phénoménologique de la mémoire », le deuxième «couple oppositionnel » constitué de la paire « évocation/recherche » (Ricœur, $2003: 32$ ) peut s'avérer particulièrement fécond afin de repérer ce qui se trouve en jeu dans l'anamnèse paradoxale du texte hébertien. L'évocation désigne ici le surgissement d'un souvenir (sous forme de sensations ou d'images), sans que ce surgissement ne soit prémédité, en quoi il est apparenté à la mémoire involontaire, par exemple. La recherche, ou le rappel, recoupe plutôt toute une tradition ayant fait de la mémoire le siège privilégié d'une assise subjective, de la réminiscence platonicienne à I'anamnèse, ayant toutes deux à franchir les barricades dressées par l'oubli.

À ce niveau minimal de la démonstration, on peut être tenté d'établir une rapide analogie du comportement mémoriel mis en récit dans Kamouraska et Le premier jardin, dans la mesure où les deux romans font appel à un champ lexical extrêmement rapproché pour rendre compte de la frayeur provoquée par une mémoire au contenu toujours énigmatique pour le lecteur. Ainsi, Le premier jardin s'ouvre sur les retrouvailles de Flora Fontanges avec la ville de son enfance, avec laquelle « [t]out ce qu'elle espère, c'est qu'il ne se produise rien (ni heurt ni émotion) ». ( $P J$ : 13) Mais à cette peur de l'évocation susceptible d'être produite par la ville, elle va très rapidement opposer la stratégie du rappel, convoquant toutefois une matière mémorielle avant tout collective, qui a pour fonction d'entraver le retour inopiné du souvenir personnel. Ce qu'elle craint par-dessus tout est de ce fait identifié très clairement lorsqu'elle évoque le fait de «tomber en enfance »: «[U]ne petite digue qui cède dans le cerveau, et le passé surgit, dru comme le mercure, envahit le présent et le noie ». ( $P J: 39)$ C'est pourtant exactement ce qui se produira, lors d'une nuit de solitude dans sa chambre d'hôtel, et «que de grands pans de mémoire cèdent alors qu'elle est couchée dans le noir, livrée, pieds et poings liés, aux images anciennes qui l'assaillent avec force. » $(P J: 127)$ Ce vocabulaire de « digue » et de «pans » est intéressant, en ceci qu'il fait de la mémoire une «vague » dangereuse qui menace d'emporter le présent même du sujet, c'est-à-dire son identité, si elle n'est pas contenue par une force «qui retient ». On retrouve ce même vocabulaire, à quelques 
nuances près, dans le roman de 1970 .

On se souvient que Mme Rolland craint l'évocation au même titre que Flora Fontanges, même si on peut croire que l'objet de cette crainte, le souvenir de l'attente de son amant après le meurtre d'Antoine Tassy, son mari, n'a pas fait l'objet du même refoulement que I'incendie de l'orphelinat chez Flora (et la mort de sa mère en couches que ce premier trauma recouvre, comme le souligne Lori Saint-Martin [1995]). Mais dans les nuits de veille de son mari mourant, les images menacent de faire retour, ce à quoi elle oppose dans un premier temps les « défenses protectrices » de l'angoisse (Kamouraska, $1970: 7$ ). Car n'oublions pas qu'à travers la mort de M. Rolland, c'est I'homme qui la «protège » $\left(K^{12}: 24\right)$ de ces images qui s'apprêtent à disparaître. Deux réactions me semblent ainsi intéressantes à relever à travers la panique occasionnée par ce décès : la peur, dans un riche moment d'évocation, d'y jouer une nouvelle fois le premier rôle (en ne parvenant pas, par exemple, à mettre la main sur le sucre à temps pour y mettre les gouttes de l'ordonnance du médecin), et le recours spontané de Mme Rolland à ses propres enfants afin de « s'en faire un rempart. » $(K: 19)$

Dans l'un et l'autre cas, donc, c'est tout un lexique d'ouvrages de défense, « digue » ou « rempart », qui est mis à contribution afin de décrire la résistance du sujet envers les risques de l'évocation. Or, dans Le premier jardin, ce vocabulaire s'ajuste à toute une série de gestes, de stratégies d'évitement qui finissent par composer la structure même de l'identité de substitution de l'actrice.

C'est ici que l'on peut revenir à la lecture de Beckett et au rôle joué par les trois éléments que le roman lui emprunte. Car c'est une évidence, le statut d'actrice de Flora Fontanges met directement de l'avant le rôle du regard. Celle-ci se compose sciemment le visage qu'elle croit approprié au gré des circonstances; surtout, elle se plaît à incarner les figures du passé que les lieux autour d'elle évoquent, comme autant de souvenirs au carrefour d'une mémoire individuelle et collective. Au même titre que Winnie a besoin de savoir que « [q]uelqu'un [la] regarde encore. (Un temps.) Se soucie d['elle]encore » (Beckett, $1986: 60)$, Flora éprouve la nécessité de ce regard de l'autre, afin de jouer, et ce, «[m]ême s'il n'y avait qu'un seul spectateur devant elle ». ( $P J: 133)$ Or, et cette différence est de taille, cet autre ne garantit pas du tout à Flora une identité propre, mais l'assure de l'efficacité du masque derrière lequel elle camoufle le fait que sans lui, « elle n'existe plus tout à fait. » ( $P J: 49)$ On sait que 
le jeu, cette capacité d'habiter le moindre nom de femme qu'elle rencontre, a servi dès les lendemains de l'incendie de l'hospice Saint-Louis : "Cela a commencé par un nom qu'on lui a donné et qu'elle a pris et, petit à petit, elle s'est mise à ressembler à Marie Eventurel, telle qu'on désirait qu'elle soit. » ( $P J: 137)$ Selon une structure hystérique parfaite, la petite Pierrette Paul, devenue Marie Éventurel, puis Flora Fontanges, multipliera les occasions de changer de masque, dans la mesure où un autre, quel qu'il soit, est là pour en assurer la vraisemblance : «Un nom, rien qu'un nom, et ça existe déjà très fort en elle. » $(P J: 50)$ Ce faisant, en jouant lui-même le jeu de I'historien amateur ou du guide touristique, multipliant ainsi les noms de la grande et de la petite histoire de la ville de Québec, de Marie Rollet aux filles du Roi, longuement énumérées, en passant par les religieuses du $17^{\text {e }}$ siècle, jusqu'aux bonnes de la Grande-Allée qui «perdaient leur nom en rentrant en ville » et « ne gardaient plus que leur prénom » $(P J: 116)$, Raphaël va assumer un double rôle dans le théâtre de Flora : celui d'interlocuteur privilégié, qui, par sa seule présence, lui permet de multiplier les masques à volonté; de même que celui d'intermédiaire, voire de passeur, d'une histoire collective qui sert précisément de « rempart » pour sa mémoire, susceptible d'être évoquée par les lieux de son enfance; une mémoire contre une autre, afin de s'en garder.

Ainsi, deux conceptions du passé trouvent à se faire concurrence : le passé de I'histoire, chronologique et représentable, prêtant à Flora Fontanges ses visages interchangeables et faisant l'objet d'une recherche, selon la typologie de Ricœur; et le passé fuyant, traumatique, et d'ailleurs toujours agissant au sein du présent, dont il s'agit de se préserver au moyen de ces masques rendus possibles par le regard de l'autre et cette instrumentalisation de I'histoire. Que celui-ci triomphe, en bout de ligne, et permette une remémoration qui ajoute le retour du refoulé, y compris dans son incomplétude relative ${ }^{13}$, au contenu mémoriel prêté par l'histoire, cela ne fait que reconduire l'imaginaire non pas mythique, mais proprement eschatologique des commencements, et préparer Flora à de nouvelles naissances ex-nihilo, « [c]omme si le présent était un lieu flagrant et nul » (PJ : 130), suivant la citation en italique du premier poème d'Exil de Saint-John Perse.

13. Lori Saint-Martin note avec justesse que « [I]e seul trou dans le récit, c'est justement celui que constitue le secret de ses origines, unique question qui lui importe et dont la solution ne lui sera jamais révélée. » (Saint-Martin, 1995 : 670). 
En racontant l'histoire d'une identité menacée par sa propre mémoire, tout en instrumentalisant des pans de I'histoire collective afin de repousser le moment de la remémoration, Le Premier Jardin tente la difficile articulation de mémoires individuelle et collective tout aussi problématiques I'une que I'autre. Du point de vue de cette instrumentalisation, la question concerne donc l'usage de I'histoire dans l'entreprise de remémoration constamment différée racontée par le roman; pour être plus précis, le rôle attribué à l'histoire afin de repousser les images d'un trauma qui sont d'autant plus menaçantes qu'elles ne sont pas passées, mais toujours présentes et agissantes pour le sujet. Chemin faisant, Le Premier Jardin convoque une multiplicité de textes pour mener à bien cette articulation : texte de I'histoire officielle de la Nouvelle-France, et des archives ayant servi à l'écrire, du « premier jardin » à l'épisode des filles du Roi; texte biblique servant à interpréter les débuts de la colonie à partir d'une herméneutique des commencements, suivant l'utopie qui a présidé à la première mise en texte du Nouveau Monde ${ }^{14}$; textes littéraires multiples visant à problématiser le rapport au passé, en particulier la perception du temps vécu, paradoxal, dans le théâtre beckettien. Le palimpseste lisible de tous ces textes, dans le roman, conduit par le fait même à complexifier la représentation d'une temporalité qui oscille entre le re-commencement du Nouveau Monde et l'attente d'une fin constamment ajournée, « retenue » par la pérennité de I'histoire. On ne saurait mieux dire que la mort, le trou dans la surface du visible, c'est-à-dire la catastrophe ou le nom indéchiffrable à l'origine de l'aventure, ont toujours déjà eu lieu, et qu'il faut donc recommencer. 


\section{Bibliographie}

AGAMBEN, Giorgio (2000), Le temps qui reste, trad. Judith Revel, Paris, Rivages.

BECKETT, Samuel (1957), Fin de partie, suivi de Acte sans paroles, Paris, Minuit.

BECKETT, Samuel (1986) [1963/1974], Oh les beaux jours, suivi de Pas moi, Paris, Minuit.

BECKETT, Samuel (1989) [1952], En attendant Godot, Paris, Minuit.

BECKETT, Samuel (1991), Cap au pire, trad. Édith Fournier, Paris, Minuit.

BECKETT, Samuel (1995) [1951], Malone meurt, Paris, Minuit.

DUMONT, Fernand (1993), Genèse de la société québécoise, Montréal, Boréal.

FALARDEAU, Érick (1997), « Fictionnalisation de I'histoire, Le Premier Jardin d'Anne Hébert », Voix et images, $\mathrm{n}^{\circ} 66$ : 557-568, repris dans Janet M. Paterson et Lori Saint-Martin (dir.), Anne Hébert en revue, Québec/Montréal, Presses de l'Université du Québec/Voix et images, 2006 : 195-205.

HÉBERT, Anne (1970), Kamouraska, Paris, Seuil.

HÉBERT, Anne (1982), Les fous de Bassan, Paris, Seuil.

HÉBERT, Anne (1988), Le premier jardin, Paris, Seuil.

HÉBERT, Anne (1992), CEuvre poétique 1950-1990, Montréal, Boréal, coll. « Compact ».

KAFKA, Franz (2001) [1953], « Cahiers in octavo » dans Préparatifs de noce à la campagne, trad. Marthe Robert, Paris, Gallimard, coll. « L'imaginaire ».

LACAN, Jacques (1986), Le séminaire. Livre VII. L'éthique de la psychanalyse, Paris, Seuil, coll. « Champ freudien $»$.

MARCHEIX, Daniel (2005), Le mal d'origine : temps et identité dans l'œuvre romanesque d'Anne Hébert, Québec, L'instant même.

MITCHELL, Constantina T. et Paul R. CÔTÉ (1991), « Ordre et rite : la fonction du cortège dans Le premier jardin d'Anne Hébert », The French Review, vol. 64, n 3 : 451-462.

NEPVEU, Pierre (1998), Intérieurs du Nouveau Monde. Essais sur les littératures du Québec et des Amériques, Montréal, Boréal, coll. " Papiers collés ».

RICOEUR, Paul (2003), La mémoire, l'histoire, l'oubli, Paris, Seuil, coll. « Points ».

SAINT-MARTIN, Lori (1995), « Les premières mères, Le premier jardin », Voix et images, n 60 : 667-681, repris dans Janet M. Paterson et Lori Saint-Martin (dir.), Anne Hébert en revue, Québec/Montréal, Presses de l'Université du Québec/Voix et images, 2006 : 207-218.

SCHMITT, Carl (2001), Le nomos de la terre dans le droit des gens du Jus publicum europaeum, trad. Lilyane Deroche-Gurcel, Paris, Presses universitaires de France, coll. « Léviathan »; révisé, présenté et annoté par Peter Haggenmacher.

TAUBES, Jacob (1999), La théologie politique de Paul. Schmitt, Benjamin, Nietzsche et Freud, trad. Mira Köller et Dominique Séglard, Paris, Seuil, coll. « Traces écrites ».

TAUBES, Jacob (2009), Eschatologie occidentale, trad. Raphaël Lellouche et Michel Pennetier, Paris, Éditions de l'éclat, coll. «Philosophie imaginaire ». 
ŽIŽEK, Slavoj (2010), « Le cogito en littérature : Descartes avec Beckett », dans Quatre variations philosophiques. Sur thème cartésien, trad. Anne Parisot, Meaux, Germina, coll. « Cercle de philosophie » : 129163.

ŽlŽEK, Slavoj (2011), Le plus sublime des hystériques. Hegel avec Lacan, Paris, Presses universitaires de France, coll. "Travaux pratiques ». 